\title{
Desenvolvimento de uma Aplicação Web para Auxílio na Abordagem do tema Cyberbullying no Ensino Fundamental
}

\author{
Ariel Carlos Peres Vieira \\ Universidade Federal da Grande Dourados - UFGD \\ arielvieira65@gmail.com
}

\begin{abstract}
The paper describes a Web application focused on the theme cyberbullying. The application aims to help educators in the approach of cyberbullying in primary schools, through a non-linear interactive narrative. The interaction of students with the application makes the learning process more attractive and interesting. The tool was evaluated based on an usability test, aiming to analyze aspects of user interaction with the system. The results show that the application complies with what is proposed, and that there was satisfaction on the part of the evaluation participants.
\end{abstract}

\section{KEYWORDS}

Cyberbullying, Cidadania Digital, Objetos de Aprendizagem

\section{INTRODUÇÃO}

O presente trabalho permeia a cultura digital, conceito referente às relações humanas mediadas por tecnologias e comunicações por meio digital [1]. Mais especificamente, a pesquisa trabalha no âmbito da cidadania digital, que aborda o uso responsável da tecnologia por parte dos indivíduos, contemplando, assim, o tema aqui tratado: o cyberbullying. Este, pode ser definido como uma manifestação de agressão ou assédio moral, que ocorre por meio de recursos tecnológicos modernos, principalmente através de telefones celulares e da Internet [2].

No contexto atual, o uso de recursos tecnológicos na educação tem provocado significativas mudanças no formato de se pensar o ensino e a aprendizagem e, apesar de trazer inúmeros benefícios, também traz algumas barreiras [3]. Um exemplo é que muitas vezes os materiais são desenvolvidos em grandes blocos, aspecto este que dificulta sua reutilização. Para tentar quebrar esta e outras barreiras, surgem os Objetos de Aprendizagem (OAs), podendo ser definidos como sendo qualquer recurso digital, o qual pode ser utilizado e reutilizado para o processo de ensino-aprendizagem [4].

Sendo assim, o intuito deste trabalho é desenvolver um OA, no formato de uma aplicação Web, para auxiliar educadores a abordarem o cyberbullying no âmbito do ensino fundamental, mais especificamente com crianças/adolescentes de 13 a 14 anos. A aplicação intenta oferecer uma narrativa textual não-linear, em que o usuário deve tomar decisões ao decorrer da história, escolhendo os caminhos que deverão ser seguidos pela personagem. Essa abordagem proporciona uma forma interativa de expor o tema, propiciando maior imersão dos alunos na narrativa apresentada.

O termo cultura digital e os conceitos atrelados a ele utilizados nesta pesquisa, têm como referência o Currículo de Referência em Tecnologia e Computação, desenvolvido pelo CIEB (Centro de Inovação para a Educação Brasileira) [1]. Este currículo tem como

\author{
Valguima Victoria Viana Aguiar Odakura \\ Universidade Federal da Grande Dourados - UFGD \\ valguima.odakura@gmail.com
}

objetivo auxiliar gestores e professores na implementação da Base Nacional Comum Curricular (BNCC) contemplando os temas de tecnologia e computação.

O trabalho está organizado como se segue: na seção 2 são apresentados trabalhos desenvolvidos na área; na seção 3 são expostos os conceitos de objetos de aprendizagem, narrativas não-lineares, hipertexto/hipermídia e cyberbullying; na seção 4 o processo de desenvolvimento da aplicação é apresentado; na seção 5 é relatado o processo de avaliação da aplicação; por fim, na seção 6 as considerações finais e trabalhos futuros são expostos.

\section{TRABALHOS RELACIONADOS}

Através do trabalho de busca por referências na área, a seguir são relatados alguns dos principais trabalhos os quais serviram como fonte de informação e inspiração.

O projeto Narrativas Cyberbullying [5] consiste na elaboração de uma narrativa digital abordando a temática de crimes relacionados às práticas de cyberbullying, fornecida através de uma aplicação Web para divulgação de relatos e materiais propícios para o estudo e conscientização do tema. A narrativa desenvolvida pelos autores, relata situações escolares de uma adolescente chamada Ana, nela o leitor tem a possibilidade de interagir com a história, tomando decisões que impactarão diretamente o seu desfecho. O site fornece a experiência de uma leitura dinâmica proporcionada pelo uso de hiperlinks, dando ao leitor diferentes possibilidades de caminhos a serem seguidos.

No trabalho de Viganó [6] são relatados o desenvolvimento e a aplicação de uma narrativa interativa digital, com intuito de investigar o potencial do uso deste formato narrativo na prevenção do suicídio entre o público adolescente. A narrativa, intitulada "A $\mathrm{Mu}$ dança", foi construída por meio do software twine, uma ferramenta open-source para o desenvolvimento de narrativas não-lineares por meio de hipertextos [7]. Como forma de validação, foram aplicados dois questionários à dois grupos de foco distintos. No primeiro, composto por jovens de uma turma universitária com idades entre 18 e 23 anos, verificou a relevância e o conhecimento a respeito do tema. O segundo questionário, aplicado à um grupo composto por profissionais da área da saúde mental, buscou analisar o conteúdo, o formato e a legitimidade do modelo. A autora conclui que os objetivos do trabalho foram alcançados, e que os resultados obtidos pela interação com a narrativa mostraram sucesso no auxílio para identificar sinais de alerta e que comportamento deve ser adotado na hora de amparar alguém em uma situação delicada.

Por fim, Sobreira et al [8] descrevem um trabalho desenvolvido com duas turmas do 5o ano de uma escola municipal, com objetivo 
de estimular a produção de textos com qualidade, através da utilização de recursos tecnológicos para a construção de uma narrativa não-linear. Inicialmente, como forma de contextualização e familiarização com o tema contos maravilhosos, os alunos estudaram algumas obras relacionadas a temática. Após este estudo, os estudantes foram orientados a reescreverem algumas das histórias contadas, utilizando laptops educacionais, o que proporcionou recursos que auxiliaram na escrita, como revisores de texto. A turma, então, foi instruída pela professora a produzir um conto maravilhoso coletivo, participando com opiniões que respeitassem as características do tema. Com a história finalizada, foi proposta a produção de uma narrativa não-linear com base no texto já criado, então, através de discussões em sala os alunos foram decidindo os caminhos que a narrativa poderia tomar, formando assim uma nova história, mais interativa e atrativa. A pesquisa traz diversas conclusões positivas a respeito dos recursos utilizados durante o trabalho e deixa evidente que a tecnologia pode ser forte aliada para a melhoria na produção escrita e para o oferecimento de uma leitura mais dinâmica.

Observa-se que todos os trabalhos aqui apresentados, se relacionam através da aplicação de narrativas não-lineares com intuito de informar ou melhorar o aprendizado de determinado público sobre determinado assunto, além de apresentarem resultados positivos, expondo diversos benefícios propiciados pela união de uma forma interativa de comunicação e de recursos tecnológicos.

Este trabalho tem forte inspiração no projeto Narrativas Cyberbullying, abordando o tema do cyberbullying através de uma narrativa não-linear, porém visa atingir o público juvenil dos últimos anos do ensino fundamental, e busca expor os conceitos envolvidos no desenvolvimento da pesquisa. Tanto Viganó [6] quanto Sobreira et al [8] demonstraram resultados positivos ao utilizarem o modelo narrativo não-linear, o que ressalta a efetividade dessa abordagem.

\section{FUNDAMENTAÇÃO TEÓRICA}

Nesta seção são expostos os principais fundamentos teóricos utilizados para o desenvolvimento do trabalho, onde: na seção 3.1 são discutidos alguns dos impactos causados no processo de ensinoaprendizagem, pelo avanço e uso de recursos tecnológicos atuais; na seção 3.2 são dadas as definições de narrativas não-lineares e de hipertexto/hipermídia, relacionando ambos os conceitos e expondo como esta relação pode proporcionar uma ferramenta dinâmica e interativa para auxílio no processo de ensino-aprendizagem; e a seção 3.3 define o conceito de cyberbullying, assim como discorre sobre as principais características atreladas ao mesmo.

\subsection{Uso de recursos tecnológicos e objetos de aprendizagem na educação}

O uso de recursos tecnológicos na educação tem provocado significativas mudanças no formato de se pensar o ensino e a aprendizagem, em especial no contexto atual, em que a Internet proporciona uma gama de possibilidades que podem ressignificar o processo educacional como um todo [3].

A quantidade de materiais educacionais presentes na Internet vem crescendo cada vez mais, podendo isto ser visto como uma grande vantagem, tanto para os educadores quanto para os educandos. Porém, em contrapartida a isso, a grande quantidade de conteúdo exposta ao usuário, em uma busca, pode trazer dificuldades na hora de encontrar ou selecionar aquilo que atenderia as suas necessidades [3]. Outra barreira que dificulta o processo da utilização desses materiais, apontada por Braga et al [3], é que muitos são desenvolvidos em grandes blocos, isso limita sua capacidade de reaproveitamento, já que pode impossibilitar a fragmentação em blocos menores para serem utilizados em diferentes situações ou contextos educacionais.

Diante deste contexto apresenta-se o conceito de Objetos de Aprendizagem (OAs), como qualquer recurso digital, como textos, animação, vídeos, imagens, aplicações $W e b$, entre outros, que destinam-se a apoiar o processo de ensino-aprendizagem [3,4].

Braga et al [3] nos apresentam duas perspectivas relacionadas as características de um OA: a pedagógica e a técnica. Sob a perspectiva pedagógica, temos uma concepção de objetos que facilitem e auxiliem o trabalho de professores e estudantes, este contexto leva em conta alguns aspectos fundamentais: interatividade, autonomia, cooperação, cognição, e afetividade [3,9]. Já sob a perspectiva técnica, temos aspectos relacionados à questões tecnológicas: confiabilidade, interoperabilidade, armazenamento, acessibilidade, reusabilidade, agregação, etc. [3, 9].

Sendo assim, a aplicação de OAs digitais na educação, além de auxiliar o educador a utilizar recursos tecnológicos em diferentes contextos de ensino, proporciona aos educandos uma dinâmica diferente à dada pela forma tradicional de ensino, podendo facilitar a assimilação de conhecimento, favorecendo assim o aprendizado.

\subsection{Narrativas não-lineares e hipertexto/hipermídia}

Uma narrativa não-linear apresenta diferentes linhas narrativas, o que proporciona uma gama de finais alternativos, consequentemente diferentes, e o que determina qual destes finais será alcançado são os caminhos tomados ao longo da narrativa $[6,10]$.

Ao se pensar em obras narrativas não-lineares, comumente o que se imagina são os jogos. Os RPGs (Role Playing Games) são ótimos exemplos de jogos que exploram um formato de narrativa não-linear. As escolhas feitas pelos jogadores no decorrer do jogo, afetam diretamente a narrativa, podendo fazer com que esta mude e tome caminhos diferentes [11].

O conceito de não-linearidade atrelado a forma de passar informações não é algo novo, podendo ser observado desde antes do advento da tecnologia como a conhecemos hoje, em campos como a arte, entretenimento, ciência e comunicação [10]. Porém, em um contexto atual, levando em conta a evolução dos meios digitais, nota-se um grande desenvolvimento de uma forma digitalizada de expor conteúdos, que depende do entrelace das informações e requer uma participação mais efetiva dos usuários [10].

Este novo formato de linguagem, que faz uso de recursos computacionais, foi proporcionado pelo que conhecemos hoje como hipertexto/hipermídia [10].

O hipertexto eletrônico é grande facilitador da interconexão, navegação e acesso imediato à conteúdos na Internet, sendo que o usuário tem a liberdade de decidir por quais caminhos navegará, formando assim uma estrutura única, o que consequentemente permite que o processo não possua uma ordem específica [6, 12]. 
Portanto, o conceito de hipertexto/hipermídia, em meios digitais, atrelado à popularização da Internet, proporciona uma poderosa ferramenta para a apresentação e utilização das narrativas nãolineares na educação, podendo resultar em maior dinamicidade e interatividade no processo de ensino-aprendizagem.

\subsection{Cyberbullying: definição e características}

Segundo Slonje e Smith [2], o cyberbullying pode ser definido como uma manifestação de agressão ou assédio moral, que ocorre por meio de recursos tecnológicos modernos, principalmente através de telefones celulares e da Internet.

Pelo fato do cyberbullying não depender de uma ambiente físico para acontecer, existem aspectos importantes que devem ser observados e que o diferem do bullying tradicional, alguns deles são: um ambiente virtual possibilita uma perseguição constante que pode chegar a 24 horas por dia, nos sete dias da semana; nos ataques presenciais de bullying a repetição é marcante, já no cyberbullying essa característica pode não existir, pois as tecnologias atuais proporcionam enorme rapidez na propagação de mensagens, fazendo com que em pouco tempo elas possam atingir uma quantidade de pessoas enorme, potencializando o pavor, a vergonha e a humilhação sofrida pela vítima; outro ponto importante é que a relação desigual de poder que caracteriza o bullying pode não existir no cyberbullying, crianças e adolescentes podem atacar professores e o menino franzino e tímido que apanha dos valentões pode inverter os papéis em um ambiente virtual; e no cyberbullying o autor dos ataques pode se esconder no anonimato, isso gera um aumento na incidência e na crueldade dos ataques [13].

Quanto às formas de ataque no cyberbullying, elas são inúmeras: ataques por mensagens de texto ou voz no celular; filmagens ou fotografias que podem ser expostas na Internet; criação de perfil falso em redes sociais; e adulteração de fotos são apenas alguns exemplos [13].

\section{DESENVOLVIMENTO}

O processo de desenvolvimento da aplicação Web se deu através da metodologia de desenvolvimento de OAs denominada INTERA (Inteligência, Tecnologias Educacionais e Recursos Acessíveis), a qual se baseia em processos de desenvolvimento de software e no modelo ADDIE para o desenvolvimento de conteúdos instrucionais. Desse modo, a metodologia INTERA contempla tantos os aspectos educacionais quanto tecnológicos do OA [14].

Essa metodologia deve ser vista como um conjunto de processos para o desenvolvimento de qualquer tipo de conteúdo digital utilizado no processo de ensino-aprendizagem [14].

A metodologia INTERA proposta por Braga et al [14] contém os seguintes componentes:

- Fases: Normalmente são sequenciais e são definidas pela entrega de algum componente do OA, a metodologia possui as fases inicial, intermediária e de transição;

- Papéis: Descrevem as funções de cada pessoa no projeto e como elas devem se comportar. Analista, conteudista, gerente de projetos, demandante, designer de interface, designer instrucional, equipe de desenvolvimento e equipe de teste são considerados papéis na metodologia INTERA;
- Artefatos: São os dados que contém informações geradas pela equipe, como documentos e código fonte, por exemplo;

- Etapas: As etapas relacionam atividades em comum a serem executadas ou desenvolvidas em um determinado período de tempo.

Em relação as etapas, tem-se:

- Contextualização, em que é definido o público-alvo, objetivo de aprendizagem, modalidade de ensino, cenário e contexto de aplicação;

- Requisitos, etapa em que há o levantamento de características técnicas e pedagógicas;

- Arquitetura, são definidas as tecnologias e padrões que serão empregados no desenvolvimento do OA;

- Desenvolvimento, a partir dos resultados das fases anteriores é desenvolvido o OA, o guia de utilização e são definidos os direitos autorais;

- Teste e Qualidade, etapa de validação e verificação de qualidade do OA desenvolvido;

- Disponibilização, consiste na disponibilização do OA, normalmente em um repositório;

- Avaliação, tem como objetivo avaliar a aprendizagem a partir da aplicação do OA;

- Gestão de projetos, permeia as demais etapas e visa coordenar, acompanhar o desenvolvimento e gerenciar o cronograma;

- Ambiente, responsável pelo controle do ambiente técnico de desenvolvimento do OA.

A equipe de trabalho foi composta por uma professora da área de computação ocupando o papel de gerente de projeto, um acadêmico também de computação com papel de desenvolvedor e conteudista e uma equipe de testes contendo 12 pessoas.

$\mathrm{Na}$ etapa de contextualização foi feita uma pesquisa sobre o tema abordado no trabalho, produzindo como artefatos os dados e embasamento para o desenvolvimento do OA. Nesta etapa foram definidos o público-alvo, os objetivos e o contexto de aplicação do OA a ser desenvolvido. Sendo o público-alvo correspondente à estudantes dos últimos anos do ensino fundamental, mais especificamente com idades entre 13 e 14 anos. O objetivo do trabalho foi definido como sendo desenvolver um OA em formato de aplicação Web, o qual traz a tona a discussão sobre o cyberbullying em um contexto escolar.

$\mathrm{Na}$ etapa de requisitos foram realizados brainstormings com professores, acadêmicos e formados na área de computação, os quais resultaram em sugestões e ideias para a elaboração da interface da aplicação e criação das narrativas, assim como funcionalidades necessárias. Definiu-se aqui o modelo a ser seguido de narrativa não-linear e a necessidade da utilização de ilustrações, pois é um grande atrativo ao público-alvo.

Na etapa de arquitetura foram definidas as tecnologias utilizadas para o desenvolvimento, sendo a principal delas a biblioteca para desenvolvimento de aplicações Web ReactJS [15], a qual tem como base a linguagem de programação JavaScript e se utiliza de tecnologias comuns da Web como HTML e CSS.

A etapa de desenvolvimento, inicialmente, envolveu a criação da narrativa não-linear e de todos os caminhos possíveis de serem percorridos pelo usuário, na criação de personagens e das ilustrações, 
e, posteriormente, no desenvolvimento da aplicação Web contendo a narrativa. Nesta etapa foi gerado o OA, de fato ${ }^{1}$. Na Figura 1 é mostrada uma das telas da aplicação, em que é possível ver a personagem principal, Rebeca.
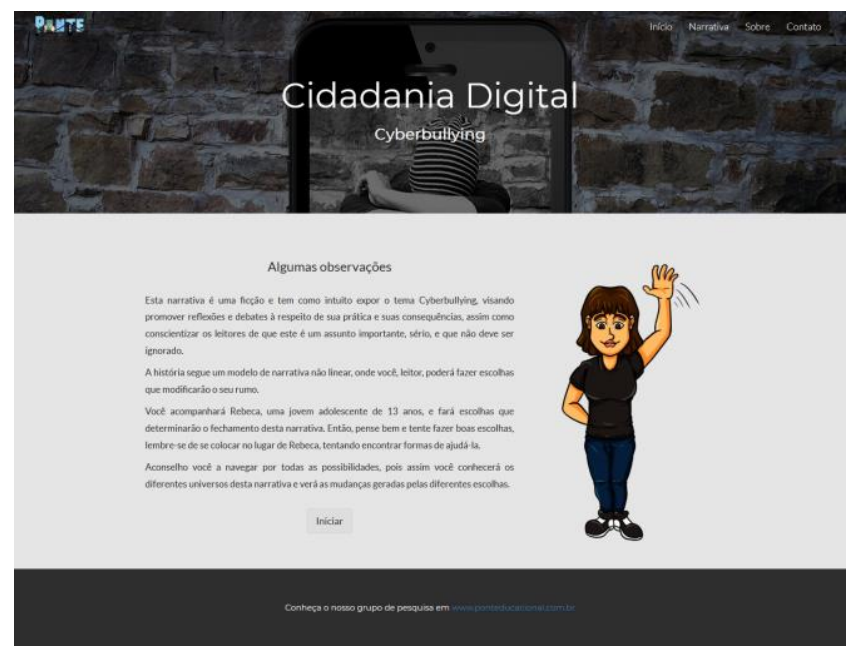

Figura 1: Exemplo de tela da aplicação desenvolvida.

Na Figura 2 é possível observar o formato geral da narrativa apresentada na aplicação. Cada página contém um trecho da história, sempre acompanhado de uma ilustração referente ao texto, e são apresentadas duas opções ao leitor, onde cada uma leva a narrativa para um caminho diferente.

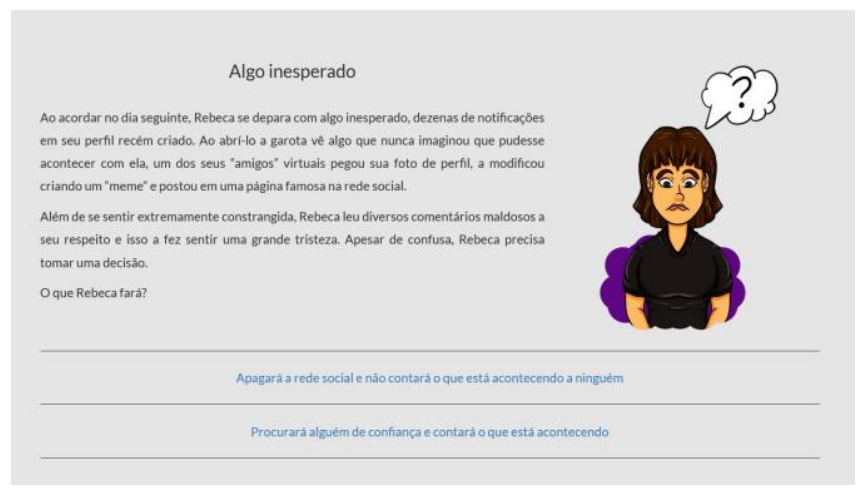

Figura 2: Exemplo de trecho da narrativa.

Na etapa de teste e qualidade foram realizados testes pela equipe de testes já descrita, os quais geraram relatórios contendo erros, assim como sugestões de melhorias que em muitos casos foram adotadas.

A etapa de disponibilização, inicialmente, se deu de forma provisória através da hospedagem da aplicação de forma gratuita em uma plataforma chamada Vercel [16]. Estuda-se a possibilidade de futuramente disponibilizar o OA em um repositório.

\footnotetext{
$1 \mathrm{O}$ projeto cyberbulling pode ser visualizado em: https://projeto-narrativacyberbullying.vercel.app/
}

A etapa de avaliação não pode ser realizada com o público-alvo do trabalho, pois esta seria feita através da aplicação do OA em uma escola pública de ensino básico, porém o período de avaliação da pesquisa coincidiu com a pandemia da COVID-19, impossibilitando a ideia inicial da avaliação. Sendo assim, optou-se pela realização de uma avaliação de usabilidade aplicada a equipe de testes, visando avaliar aspectos da interação do usuário com a aplicação. Esta avaliação é descrita de forma detalhada na seção 5 . Futuramente pretende-se levar o trabalho às escolas para concluir a ideia inicial e obter uma avaliação mais efetiva já que será realizada pelo públicoalvo.

\section{AVALIAÇÃO}

A avaliação do site segue o método de teste de usabilidade, o qual visa avaliar a qualidade da interação do usuário com a aplicação, identificando assim problemas e pontos de melhoria [17].

Para a avaliação foi aplicado um formulário à equipe de teste, contendo 4 tarefas a serem realizadas na aplicação Web e 13 questões, referentes a usabilidade, a serem respondidas. Cada tarefa teve como objetivo avaliar um ou mais aspectos da interação do usuário com a aplicação. Após a realização das tarefas, cada participante foi orientado a responder o questionário, o qual teve como objetivo coletar a opinião sobre a experiência de cada um ao realizar as tarefas propostas. O questionário foi desenvolvido com base em uma escala likert, consistindo em uma série de questões em que os usuários escolhem uma dentre cinco alternativas, sendo elas: concordo totalmente; concordo parcialmente; não concordo, nem discordo; discordo parcialmente e discordo totalmente [18].

Os resultados coletados através da escala likert são analisados por meio da atribuição de valores para cada item. Neste trabalho, o valor utilizado variou de 1 (discordo totalmente) à 5 (concordo totalmente).

A seguir são apresentadas as tarefas e questões desenvolvidas para a avaliação, assim como os resultados coletados com o formulário:

Tarefas:

(1) Percorra um caminho completo da narrativa.

(2) Retorne ao início da narrativa e percorra um caminho diferente.

(3) Encontre o e-mail para contato com o responsável pelo site.

(4) Caso tenha executado as tarefas em um desktop/notebook, execute-as novamente (se possível) em um smartphone/tablet.

\section{Questões:}

(1) O site apresenta boa legibilidade.

(2) As ilustrações apresentam boa qualidade e contribuem para deixar a narrativa mais atrativa e interessante.

(3) Consegui percorrer diferentes caminhos sem dificuldades.

(4) Consegui encontrar um meio de contato sem dificuldades.

(5) Consegui encontrar o que procurava sem dificuldades.

(6) Não é necessário conhecimento específico sobre algum assunto para se utilizar o site.

(7) O site possui uma interface agradável.

(8) O site é auto descritivo, ou seja, as informações contidas informam o propósito do site. 
(9) O site apresentou comportamento normal, sem nenhum comportamento inesperado durante a realização de alguma tarefa.

(10) Você se sentiu satisfeito em realizar as tarefas no site.

(11) Caso tenha executado as tarefas em ambos os dispositivos: o site é responsivo, ou seja, se adéqua à diferentes dispositivos.

(12) Encontrou algum problema no site? Relate aqui.

(13) Sugestões para melhoria do site? Compartilhe conosco.

Em relação a aplicação do teste de usabilidade, pode-se visualizar a área de atuação dos participantes na Figura 3, em que é apresentado o percentual de participantes (equipe de testes), por área de atuação. Todas as avaliações são de extrema importância, porém vale ressaltar que $75 \%$ dos participantes são das áreas de Computação, Educação, Psicologia e Estudante do ensino médio, as quais estão mais ligadas à pesquisa desenvolvida.

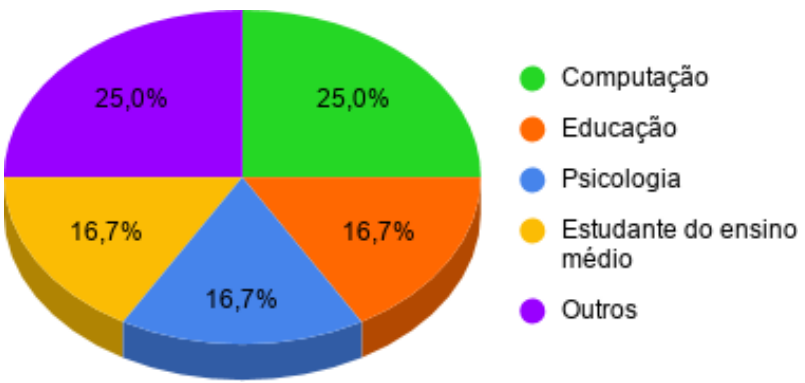

\section{Figura 3: Participantes da avaliação por área de atuação.}

Na Figura 4 são mostrados quais dispositivos foram utilizados pelos participantes para a realização das tarefas propostas. Este dado é importante para avaliar aspectos de responsividade da aplicação. Como pode ser observado, $50 \%$ dos participantes realizaram as tarefas tanto em Desktop/Notebook, quanto em Smartphone/Tablet.

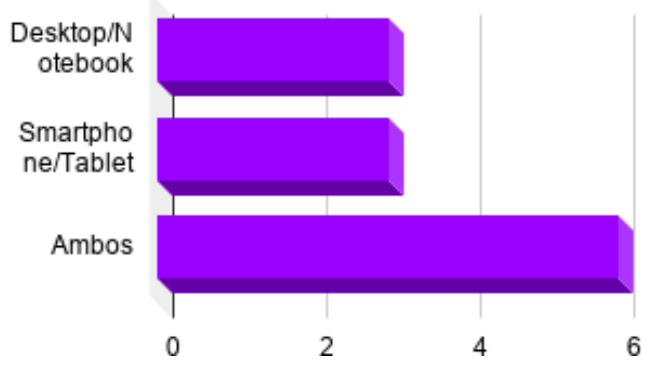

Figura 4: Dispositivos utilizados para a realização das tarefas.

Em relação as questões do teste, na Figura 5 são expostos os resultados obtidos para cada uma das 11 questões objetivas presentes no formulário de avaliação. Nota-se que para a maioria das questões os resultados foram positivos, significando que, em geral, os participantes do teste se sentiram satisfeitos em relação a interação com a aplicação Web.

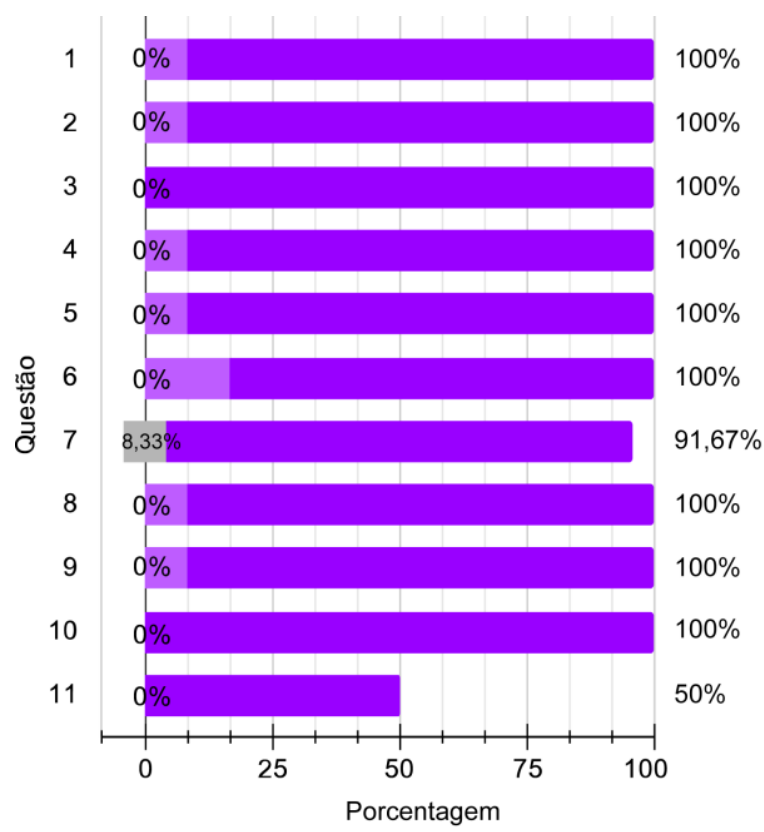

Concordo parcialmente Concordo totalmente

Discordo parcialmente Discordo totalmente

Não concordo, nem discordo

Figura 5: Resultado para cada questão objetiva.

Para analisar o critério de responsividade, considerando as respostas da questão 11, na Figura 5, é possível notar que 6 participantes, ou seja, $50 \%$, consideraram que a aplicação atende ao critério de responsividade. Vale ressaltar, que este valor de $50 \%$ equivale a todos os participantes aptos a avaliarem este critério, ou seja, os que utilizaram ambos os tipos de aplicativos, conforme Figura 4.

Além das questões objetivas, o formulário contemplou duas questões abertas e não obrigatórias. A questão 12 teve intuito de coletar o relato de possíveis problemas encontrados pelos participantes durante a execução das tarefas de forma mais detalhada e específica. Seguem as respostas obtidas:

- "Não foi encontrado nenhum problema no site."

- "Não, sem problemas."

- "Nenhum."

- "Não. Foi muito fácil encontrar os caminhos."

Como pode ser observado, os participantes que responderam à essa questão não relataram problemas, o que corrobora com os resultados obtidos através das questões objetivas.

A questão 13 buscou coletar sugestões para melhoramento da aplicação. Dentre as respostas obtidas, destacam-se as seguintes: 
- "No celular, na hora de mudar a página, a história começa no fim da página, o ideal é na página seguinte já ir direto para a parte de cima."

- "Nas próximas versões seria interessante um botão de voltar ao ponto anterior, isso facilitaria a navegação pelos caminhos."

- "Uma coisa que ficou confusa para mim, talvez tenha sido explicado em algum momento e não percebi é a questão das linhas de eventos, em algumas escolhas ela mora com a tia em outras não, caso o usuário opte por explorar todas as possibilidades ele pode ficar confuso com isso. No restante do site, pra mim está tudo ok."

O primeiro item relata a necessidade de retorno ao início das páginas após a mudança das mesmas. De fato é um aspecto relevante para a experiência do usuário, principalmente em dispositivos com a telas pequenas, portanto é pretendida a implementação desta funcionalidade.

O segundo item foi discutido durante o desenvolvimento, mas optou-se pela não utilização do recurso de voltar a página pelo fato de que a narrativa se passa em diferentes cenários, dependendo das escolhas do usuário, portanto a opção de voltar poderia tornar a história confusa, já que seria possível misturar linhas diferentes da narrativa, de forma muito fácil. Para exemplificar este ponto, há um momento na narrativa em que uma das opções dadas ao usuário leva a narrativa por uma linha em que a personagem principal mora com a sua tia, a outra opção muda a linha da narrativa, e nesta a personagem mora com seus pais. Desta forma, acredita-se que a adoção de um botão de voltar poderia tornar a narrativa confusa e consequentemente proporcionar uma experiencia ruim ao usuário.

O terceiro item relata exatamente a confusão em relação às diferentes linhas da narrativa descrita no parágrafo anterior. Para minimizar as chances deste problema acontecer, na introdução da história é dito que a mesma se passa em diferentes cenários, de forma a deixar o usuário ciente de que a narrativa possui diferentes linhas e que há mudanças significativas entre elas.

Os resultados do teste de usabilidade apontam que a aplicação desenvolvida atingiu o primeiro passo, de muitos, para que possa ser utilizada de forma satisfatória em um contexto real. Vale ressaltar que apesar de não ter sido feita uma avaliação pedagógica, participaram do teste professores e psicólogos, que consideraram a aplicação adequada para o tema e faixa etária. Avaliações posteriores em ambiente escolar irão esclarecer as questões pedagógicas da aplicação desenvolvida.

\section{CONSIDERAÇÕES FINAIS}

O presente trabalho teve como objetivo o desenvolvimento de uma aplicação Web, a qual fornecesse um OA para auxílio na abordagem do tema cyberbullying com estudantes dos últimos anos do ensino fundamental, a primeira etapa de validação trouxe resultados positivos e satisfatórios para que os próximos passos possam ser dados. Os testes de usabilidade mostram que a aplicação atende de forma satisfatória aos requisitos avaliados.

É necessário ressaltar que a avaliação não pôde ser realizada com o público-alvo e em ambiente escolar, devido ao fechamento das escolas durante a pandemia da COVID-19. Sendo assim, como trabalho futuro sugere-se a aplicação de uma avaliação neste sentido, considerando estudantes e professores, buscando avaliar aspectos da efetividade da aplicação do OA ao público e ambiente propostos.

Acredita-se que o trabalho possa contribuir para o processo de ensino-aprendizagem das seguintes formas:

- Fornecendo aos educadores uma ferramenta para a abordagem, em escolas, de um tema relevante nos dias atuais, com a crescente popularização da Internet e principalmente das redes sociais;

- Fornecendo aos estudantes do ensino básico uma forma interativa e mais atrativa de conhecer o tema do cyberbullying e algumas das consequências que sua prática pode gerar;

- Trazendo à tona a discussão e a reflexão acerca de questões envolvidas com o tema do cyberbullying, em um ambiente escolar.

Portanto, levando em conta as limitações já citadas, considera-se que o objetivo primário do desenvolvimento da aplicação atendendo aos requisitos de usabilidade foi alcançado e espera-se que futuramente a mesma seja aplicada em um ambiente real de forma a contemplar uma avaliação pedagógica, e posteriormente contribuir, na prática, para o processo de ensino-aprendizagem.

\section{ACKNOWLEDGMENTS}

Agradecimentos à UFGD através do Programa Institucional de Bolsas de Iniciação Científica (PIBIC) que possibilitou o desenvolvimento deste trabalho.

\section{REFERÊNCIAS}

[1] CIEB. Currículo de Referência em Tecnologia e Computação. https://curriculo. cieb.net.br/, 2019. [online: acesso em 09-setembro-2019].

[2] Robert Slonje and Peter K Smith. Cyberbullying: Another main type of bullying? Scandinavian journal of psychology, 49(2):147-154, 2008.

[3] Juliana Braga et al. Objetos de aprendizagem volume 1: introdução e fundamentos. Santo André: Editora da UFABC, 2014.

[4] David A Wiley et al. Connecting learning objects to instructional design theory: A definition, a metaphor, and a taxonomy. The instructional use of learning objects, 2830(435):1-35, 2000.

[5] Narrativas Cyberbullying. Narrativas Cyberbullying. https: //narrativascyberbullying.wordpress.com/, 2015. [online: acesso em 21outubro-2019].

[6] Mariana Ruaro Viganó. Narrativas interativas digitais na prevenção do suicídio, 2019. Trabalho de Conclusão de Curso. Universidade Caxias do Sul.

[7] Twine. Twine. https://twinery.org/, 2009. [online: acesso em 15-setembro-2019].

[8] Elaine Silva Rocha Sobreira, Maria Cristina Nunes, and Sandra Benedita Morassi. Produzindo histórias não lineares: um incentivo à produção escrita e leitura, através do uso contextualizado da tecnologia. In Anais do Workshop de Informática na Escola, volume 1, page 160, 2013.

[9] Carla Cristina Lui Dias, Avanilde Kemczinski, Sérgio Vinícius de Sá Lucena, Jhônatan Ferlin, and Marcelo da Silva Hounsell. Padrões abertos: aplicabilidade em objetos de aprendizagem (oas). In Brazilian Symposium on Computers in Education (Simpósio Brasileiro de Informática na Educação-SBIE), volume 1, 2009.

[10] Karla Patriota and J Cunha. Interatividade, imersão e leitura não-linear: Os novos meios e as novas linguagens. Trabalho apresentado ao NP de Tecnologias da Informação e da Comunicação, do VI Encontro dos Núcleos de Pesquisa da Intercom, Brasília, 2006.

[11] André Daniel Hayashi. O comportamento caórdico dos jogos de rpg e a aprendizagem. Rev. PEC, Curitiba, 3(1):59-66, 2002.

[12] George P Landow. Hypertext 3.0: Critical theory and new media in an era of globalization. JHU Press, 2006.

[13] Maria Tereza Maldonado. Bullying e cyberbullying: o que fazemos com o que fazem conosco. São Paulo: Moderna, 2011.

[14] Juliana Cristina Braga, Edson Pimentel, and Silvia Dotta. Metodologia intera para o desenvolvimento de objetos de aprendizagem. In Brazilian Symposium on Computers in Education (Simpósio Brasileiro de Informática na Educação-SBIE), volume 24, page 306, 2013.

[15] React. ReactJS. https://pt-br.reactjs.org/, 2020. [online: acesso em 7-novembro2020].

[16] Vercel. Vercel. https://vercel.com/, 2020. [online: acesso em 7-novembro-2020]. 
XII Computer on the Beach

7 a 9 de Abril de 2021, Online, SC, Brasil

Vieira et al.

[17] Lais Machado, Evelise Pereira Ferreira, and Lizandra Garcia Lupi VERGARA Métodos de avaliação de usabilidade: Características e aplicações. 30. CONEPROSUL, Joinville. 3o. CONEPROSUL, 2014.

[18] Bernardo Aguiar, Walter Correia, and Fábio Campos. Uso da escala likert na análise de jogos. Anais do X Simpósio Brasileiro de Games e Entretenimento Digital, 7(09), 2011. 